\title{
Spectral Expansions for Asian (Average Price) Options
}

\author{
Vadim Linetsky \\ Department of Industrial Engineering and Management Sciences, McCormick School of Engineering and Applied Sciences, \\ Northwestern University, 2145 Sheridan Road, Evanston, Illinois 60208, linetsky@iems.northwestern.edu
}

\begin{abstract}
Arithmetic Asian or average price options deliver payoffs based on the average underlying price over a prespecified time period. Asian options are an important family of derivative contracts with a wide variety of applications in currency, equity, interest rate, commodity, energy, and insurance markets. We derive two analytical formulas for the value of the continuously sampled arithmetic Asian option when the underlying asset price follows geometric Brownian motion. We use an identity in law between the integral of geometric Brownian motion over a finite time interval $[0, t]$ and the state at time $t$ of a one-dimensional diffusion process with affine drift and linear diffusion and express Asian option values in terms of spectral expansions associated with the diffusion infinitesimal generator. The first formula is an infinite series of terms involving Whittaker functions $M$ and $W$. The second formula is a single real integral of an expression involving Whittaker function $W$ plus (for some parameter values) a finite number of additional terms involving incomplete gamma functions and Laguerre polynomials. The two formulas allow accurate computation of continuously sampled arithmetic Asian option prices.
\end{abstract}

Subject classifications: finance, asset pricing: option pricing; finance, securities: Asian (average price) options;

probability, diffusion: average of geometric Brownian motion, spectral theory.

Area of review: Financial Engineering.

History: Received November 2001; revision received October 2002; accepted July 2003.

\section{Introduction}

Asian (average price) options deliver payoffs based on the average underlying price or financial variable over a prespecified time period. Asian-style derivatives constitute an important family of derivative contracts with a wide variety of applications in currency, equity, interest rate, commodity, energy, and insurance markets. To the best of our knowledge, Asian options were first introduced by Boyle and Emanuel (1980) (see Boyle 1996 for the history of Asian options).

There are a number of economic reasons why Asian options are so popular. First, when developing long-term financial projections, the corporate treasurer is often concerned with the average foreign exchange rate or commodity price realized over the accounting period. Thus, the average rate (or average price) options are a natural corporate financial risk-management tool. In addition, they are typically less expensive than standard options because the volatility of the average exchange rate or commodity price is lower than the volatility of the rate or price itself. To give a specific example, according to the recent RISK magazine interview with Microsoft treasurer Jean-Francois Heitz (Falloon 1998), Microsoft uses average rate foreign exchange options as a main tool for hedging its net revenue exposures. In 1995, 1996, and 1997, approximately 37\%, $34 \%$, and $32 \%$ of Microsoft's revenue, respectively, was collected in foreign currencies. A sizable currency exposure is the result, and Microsoft typically hedges a percentage of the planned net revenue in major currencies. For the fiscal year ending June 30,1997, the notional amount of the currency options outstanding totaled $\$ 2.1$ billion. Heitz says it is company policy to hedge the entire budgeted revenue in a given quarter. Hedging its foreign exchange exposures with average rate options helps Microsoft maintain stable earnings quarter after quarter.

A second class of applications exploits the fact that, while it may be possible for a large market participant to manipulate the price of a thinly traded commodity on any given day, it is much harder to manipulate its average price over a period of time. For this reason, many commodity contracts are Asian-style and are settled based on the average price of a commodity over a specified time period preceding contract expiration (e.g., crude oil futures and options on the NYMEX and copper and aluminium options on the London Metals Exchange). For the same reason, many corporate mergers and takeovers are also average price contingent (e.g., the mergers of Dow Chemical with Marion Laboratories and Rhone-Poulenc with the Rhorer Group).

Accurate pricing of Asian options is an important practical problem in financial engineering. This problem raises several interesting methodological issues. First, these options are path-dependent. The price of an Asian option at any point in time is a function of both the price of the underlying asset at that time and also the average of the underlying prices up to that time. Second, the 
arithmetic average is not lognormally distributed when the underlying follows the geometric Brownian motion process assumed in the standard Black-Scholes-Merton option-pricing framework. In fact, this distribution turns out to be quite complicated to characterize analytically.

In a pioneering contribution, Yor (1992) expresses the arithmetic Asian option value as a triple integral. Unfortunately, the triple integral is difficult to evaluate numerically. As an alternative, Geman and Yor (1993) derive a closedform expression for the Laplace transform of the arithmetic Asian call-option value. Their formula can be expressed in terms of the Kummer confluent hypergeometric function (see Donati-Martin et al. 2001, Yor 2001 for further references). This Laplace transform has to be inverted numerically by applying a suitable numerical Laplace inversion algorithm (Eydeland and Geman 1995, Fu et al. 1998, Craddock et al. 2000, Shaw 2002). More recently, Dufresne (2000) obtains an alternative formula that expresses the Asian option value as an infinite series of Laguerre polynomials with each coefficient in the series given by a single integral that needs to be computed numerically (see also Dufresne 2001). To compute Asian option prices using these formulas, one either needs to compute Yor's triple integral, numerically invert Geman and Yor's Laplace transform, or compute Dufresne's infinite series of integrals.

A number of authors have suggested various analytical approximations to approximate the distribution of the average. Among them are the lognormal approximation with matched first and second moments of Turnbull and Wakeman (1992) and the reciprocal gamma approximation of Milevsky and Posner (1998). The problem with analytical approximations of this type is that no reliable error estimates are available. Monte Carlo simulation (Boyle and Emanuel 1980, Kemna and Vorst 1990, Boyle et al. 1997) and numerical partial differential equation (PDE) methods (Rogers and Shi 1995; Zvan et al. 1997; Vecer 2001, 2002; Marcozzi 2003) are the two main numerical approaches to Asian options.

In this paper, we revisit the continuously sampled arithmetic Asian option problem and take a different approach. We use an identity in law between the integral of geometric Brownian motion over a finite time interval $[0, t]$ and the state at time $t$ of a one-dimensional diffusion process with affine drift and linear diffusion. The problem of pricing Asian options is thus equivalent to the problem of pricing vanilla European options on this diffusion. We solve the latter problem by expressing option values in terms of spectral expansions associated with the diffusion infinitesimal generator. This leads us to two representations for the Asian option value. The first representation is an infinite series of terms involving Whittaker functions $M$ and $W$. The second representation is a single real integral of an expression involving Whittaker function $W$ plus (for some parameter values) a finite number of additional terms involving incomplete gamma functions and Laguerre polynomials. The two formulas can be programmed in Mathematica and Maple and allow accurate computation of continuously sampled arithmetic Asian option values. We give numerical examples accurate to 10 decimals.

The remainder of this paper is organized as follows. Section 2 describes continuously sampled arithmetic Asian options, sets up the valuation problem in the BlackScholes-Merton environment, and reduces it to the problem of pricing European options on a one-dimensional diffusion with affine drift and linear diffusion. Section 3 states the main results: the series representation (Proposition 1) and the integral representation (Proposition 2) for continuously sampled arithmetic Asian options. Section 4 contains the proof of Proposition 1 by constructing the spectral representation of the diffusion of $\$ 2$ considered on the finite interval $[0, b]$ with the killing boundary at $b<\infty$. Section 5 contains the proof of Proposition 2 by taking the limit $b \uparrow \infty$. Section 6 presents our computational results and comparisons with other approaches in the literature. Section 7 concludes the paper.

\section{Continuously Sampled Arithmetic Asian Options}

We assume that, under the risk-neutral probability measure, the underlying asset price follows a geometric Brownian motion process

$S_{t}=S_{0} e^{\sigma B_{t}+\left(r-q-\sigma^{2} / 2\right) t}, \quad t \geqslant 0$,

where $\left\{B_{t}, t \geqslant 0\right\}$ is a standard Brownian motion, $\sigma>0$ is the constant volatility, $r \geqslant 0$ is the constant risk-free interest rate, $q \geqslant 0$ is the constant dividend yield, and $S_{0}>0$ is the initial asset price at $t=0$. For $t>0$, let $\mathscr{A}_{t}$ be the continuously sampled arithmetic average price:

$\mathscr{A}_{t}=\frac{1}{t} \int_{0}^{t} S_{u} d u$.

A continuously sampled arithmetic Asian call (put) with the strike price $K>0$ and expiration date $T>0$ delivers the payoff $\left(\mathscr{A}_{T}-K\right)^{+}\left(\left(K-\mathscr{A}_{T}\right)^{+}\right)$at time $T\left(x^{+} \equiv\right.$ $\max \{x, 0\})$. Asian put and call prices are related by the well-known put-call parity relationship for Asian options (see Geman and Yor 1993, Dufresne 2000):

$$
\begin{array}{rl}
e^{-r T} & \mathbb{E}\left[\left(\mathscr{A}_{T}-K\right)^{+}\right] \\
= & e^{-r T} \mathbb{E}\left[\left(K-\mathscr{A}_{T}\right)^{+}\right] \\
& + \begin{cases}\frac{e^{-q T}-e^{-r T}}{(r-q) T} S_{0}-e^{-r T} K, & r \neq q, \\
e^{-r T}\left(S_{0}-K\right), & r=q .\end{cases}
\end{array}
$$

Thus, it is sufficient to price Asian puts. Asian call prices can be recovered by appealing to the put-call parity. So far we have discussed newly written Asian options. Suppose the Asian put option has been initiated at time zero, and we are interested in pricing it at some time $0<t<T$ 
during the option's life (seasoned Asian option). It is wellknown (Geman and Yor 1993) that the problem of pricing a seasoned Asian option can be reduced to the problem of pricing a newly written Asian option with a modified strike price. We thus limit our discussion to newly written Asian put options.

Following Geman and Yor (1993), it is convenient to standardize the problem:

$e^{-r T} \mathbb{E}\left[\left(K-\mathscr{A}_{T}\right)^{+}\right]=e^{-r T}\left(\frac{4 S_{0}}{\sigma^{2} T}\right) P^{(\nu)}(k, \tau)$,

where the function $P^{(\nu)}(k, \tau)$ is defined by

$P^{(\nu)}(k, \tau):=\mathbb{E}\left[\left(k-A_{\tau}^{(\nu)}\right)^{+}\right]$,

$A_{\tau}^{(\nu)}$ is the so-called Brownian exponential functional (see Yor 2001):

$A_{\tau}^{(\nu)}:=\int_{0}^{\tau} e^{2\left(B_{u}+\nu u\right)} d u$

and the (dimensionless) parameters $\tau, \nu$, and $k$ are

$\tau:=\frac{\sigma^{2} T}{4}, \quad \nu:=\frac{2(r-q)}{\sigma^{2}}-1, \quad k:=\frac{\tau K}{S_{0}}$.

This reduction is a consequence of the Brownian scaling property: $\sigma B_{t} \stackrel{\text { (law) }}{=} 2 B_{\left(\sigma^{2} t\right) / 4}$.

Our starting point is an identity in law for fixed $t>0$ (Dufresne 1989, 1990; Carmona et al. 1997; Donati-Martin et al. 2001):

$A_{t}^{(\nu)} \stackrel{\text { (law) }}{=} X_{t}$

where $X_{t}$ is the state at time $t$ of a one-dimensional diffusion process $\left\{X_{t}, t \geqslant 0\right\}$ on $[0, \infty)$ started at the origin $X_{0}=0$ and with the infinitesimal diffusion parameter $a(x)=2 x$, infinitesimal drift $b(x)=2(\nu+1) x+1$, and infinitesimal generator

$$
\begin{aligned}
(\mathscr{G} f)(x) & =\frac{1}{2} a^{2}(x) f^{\prime \prime}(x)+b(x) f^{\prime}(x) \\
& =2 x^{2} f^{\prime \prime}(x)+[2(\nu+1) x+1] f^{\prime}(x) .
\end{aligned}
$$

This diffusion has scale and speed densities (see Borodin and Salminen 1996, p. 17):

$$
\begin{aligned}
& \mathfrak{s}(x)=\exp \left\{-\int \frac{2 b(x)}{a^{2}(x)} d x\right\}=x^{-\nu-1} e^{1 / 2 x}, \\
& \mathfrak{m}(x)=\frac{2}{a^{2}(x) \mathfrak{s}(x)}=\frac{1}{2} x^{\nu-1} e^{-1 / 2 x},
\end{aligned}
$$

respectively. For $\nu<0$, this diffusion first appeared in Wong (1964). In this paper, we consider it for all $\nu \in \mathbb{R}$. The origin is an entrance boundary for all $\nu \in \mathbb{R}$. If started at zero, the process rapidly enters the state space $(0, \infty)$ under the influence of positive drift $(+1$ in $b(x)=$ $2(\nu+1) x+1)$. Infinity is a natural boundary, attracting for $\nu>0$ and nonattracting for $\nu \leqslant 0$. For $\nu<0$, the process has a stationary distribution with the reciprocal gamma density $\pi(x) \sim x^{\nu-1} e^{-1 /(2 x)}$.

To prove (7), we note that the process $\left\{X_{t}=e^{2\left(B_{t}+\nu t\right)}\right.$ $\left.\times \int_{0}^{t} e^{-2\left(B_{u}+v u\right)} d u, t \geqslant 0\right\}$ solves the stochastic differential equation $d X_{t}=\left[2(\nu+1) X_{t}+1\right] d t+2 X_{t} d B_{t}$ with the initial condition $X_{0}=0$ (this is immediate using Ito's formula) and, by time reversal, $X_{t}=\int_{0}^{t} e^{2\left(B_{t}-B_{u}\right)+2 \nu(t-u)} d u \stackrel{\text { (law) }}{=}$ $\int_{0}^{t} e^{2\left(B_{s}+\nu s\right)} d s=A_{t}^{(\nu)}$. The identity in law (7) was applied to the valuation of Asian options by Donati-Martin et al. (2001). To compute the Asian call price, these authors observe that $\mathbb{E}\left[\left(A_{\tau}^{(\nu)}-k\right)^{+}\right]=\mathbb{E}\left[\left(X_{\tau}-k\right)^{+}\right]$. They then compute the resolvent kernel of the diffusion $X$ (Theorem 3.1) and, on integration with $(x-k)^{+}$, obtain the Laplace transform of $\mathbb{E}\left[\left(X_{\tau}-k\right)^{+}\right]$with respect to $\tau$; i.e., $\mathbb{E}\left[\left(X_{\tau_{\alpha}}-k\right)^{+}\right]$ with $\tau_{\alpha}$-a random exponential time with parameter $\alpha>0$ and independent of $B$ (Corollary 3.1 in Donati-Martin et al. 2001). This gives an alternative derivation of the Geman and Yor (1993) Laplace transform result. To recover $\mathbb{E}\left[\left(X_{\tau}-k\right)^{+}\right]$for fixed $\tau$ and, hence, the Asian call-option price, one then needs to invert the Laplace transform with respect to $\alpha$. Several authors cited in the introduction apply a number of numerical Laplace transform inversion algorithms to this problem.

In this paper, we take a different approach. We develop spectral expansions to compute $\mathbb{E}\left[\left(k-X_{\tau}\right)^{+}\right]$for fixed $\tau$ without using Laplace transforms. These spectral expansions are associated with the infinitesimal generator $\mathscr{G}$. Note that, in contrast with Geman and Yor (1993) and Donati-Martin et al. (2001), we work with puts rather than calls because $(k-x)^{+}$is in $L^{2}([0, \infty), \mathfrak{m})$ ( $\mathfrak{m}$ is the speed density of the diffusion $X$ given by Equation (9)), while $(x-k)^{+}$is not. We develop spectral expansions for puts, and price calls via the put-call parity result (3). For spectral theory of one-dimensional diffusions, we refer the reader to Linetsky (2004d) and references therein.

In addition to $P^{(\nu)}(k, \tau)=\mathbb{E}\left[\left(k-X_{\tau}\right)^{+}\right]$, we also consider up-and-out puts on the diffusion $X$. For $b>k$, consider a function

$P_{b}^{(\nu)}(k, \tau):=\mathbb{E}\left[\mathbf{1}_{\left\{\mathscr{T}_{b}>\tau\right\}}\left(k-X_{\tau}\right)^{+}\right]$,

where $\mathscr{T}_{b}:=\inf \left\{t \geqslant 0: X_{t}=b\right\}$ is the first hitting time of $b$ and $\mathbf{1}_{\left\{\mathscr{T}_{b}>\tau\right\}}=1(0)$ if $\mathscr{T}_{b}>\tau\left(\mathscr{T}_{b} \leqslant \tau\right)$. Our strategy is to first calculate the function $P_{b}^{(\nu)}(k, \tau)$ and then take the limit $b \uparrow \infty$ to recover the function $P^{(\nu)}(k, \tau)$.

\section{Main Results}

Spectral representations for $P_{b}^{(\nu)}(k, \tau)$ and $P^{(\nu)}(k, \tau)$ will be expressed in terms of several classical special functions: first and second Whittaker functions $M_{\kappa, \mu}(z)$ and $W_{\kappa, \mu}(z)$ (Abramowitz and Stegun 1972, p. 505; Slater 1960, pp. 9-10; Buchholz 1969, pp. 9-20), gamma function $\Gamma(z)$ (Abramowitz and Stegun 1972, p. 255), the incomplete 
gamma function $\Gamma(a, z)$ (Abramowitz and Stegun 1972, p. 260), and the generalized Laguerre polynomials $L_{n}^{(a)}(z)$ (Abramowitz and Stegun 1972, p. 773). All of these special functions are available in both Mathematica and Maple software packages.

\subsection{The Spectral Representation for $P_{b}^{(\nu)}(k, \tau)$}

Proposition 1. Let $\nu \in \mathbb{R}, \tau>0, k>0$, and $b>k$. Let $0<p_{1, b}<p_{2, b}<\cdots, p_{n, b} \uparrow \infty$ as $n \uparrow \infty$, be the positive simple zeros of the Whittaker function $W_{\kappa, \mu}(z)$ with the fixed first index $\kappa=(1-\nu) / 2$, fixed argument $z=1 /(2 b)$, and the purely imaginary second index $\mu=i p / 2, p>0$ $(i=\sqrt{-1})$. That $i s, p=p_{n, b}$ are the positive simple roots of the equation $\left(W_{\kappa, i p / 2}(z)\right.$ is a real function of $p$ because $W_{\kappa, \mu}(z)=W_{\kappa,-\mu}(z)$; Slater 1960, p. 11, Equation (1.7.11)):

$$
W_{(1-\nu) / 2, i p / 2}\left(\frac{1}{2 b}\right)=0 \text {. }
$$

Introduce the following notation:

$\xi_{n, b}^{(\nu)}:=\left.\frac{\partial}{\partial p} W_{(1-\nu) / 2, i p / 2}\left(\frac{1}{2 b}\right)\right|_{p=p_{n, b}}$.

For $\nu<0$, let $N_{\nu}(b), 0 \leqslant N_{\nu}(b) \leqslant[|\nu| / 2]+1 \quad([x]$ denotes the integer part of $x$ ) be the total number of roots of the equation

$$
W_{(1-\nu) / 2, q / 2}\left(\frac{1}{2 b}\right)=0
$$

in the interval $q \in[0,|\nu|)$ and, for $N_{\nu}(b)>0$, let $\left\{q_{n, b}\right.$, $\left.n=1, \ldots, N_{\nu}(b)\right\}$ be the corresponding roots ordered in descending order, $0 \leqslant q_{N_{\nu}(b), b}<\cdots<q_{1, b}<|\nu|$. Introduce the following notation:

$\eta_{n, b}^{(\nu)}:=-\left.\frac{\partial}{\partial q} W_{(1-\nu) / 2, q / 2}\left(\frac{1}{2 b}\right)\right|_{q=q_{n, b}}$.

For $\nu \geqslant 0$, Equation (13) has no roots in the interval $q \in[0, \nu)$ and $N_{\nu}(b) \equiv 0$.

Then, the function $P_{b}^{(\nu)}(k, \tau)$ is given by the following series $\left(\sum_{n=1}^{0} \equiv 0\right.$ by convention $)$ :

$$
\begin{aligned}
& P_{b}^{(\nu)}(k, \tau) \\
& =\sum_{n=1}^{\infty} e^{-\left(\left(\nu^{2}+p_{n, b}^{2}\right) \tau\right) / 2} \frac{p_{n, b} \Gamma\left(\left(\nu+i p_{n, b}\right) / 2\right)}{4 \xi_{n, b}^{(\nu)} \Gamma\left(1+i p_{n, b}\right)}(2 k)^{(\nu+3) / 2} \\
& \quad \times e^{-1 /(4 k)} W_{-(\nu+3) / 2,\left(i p_{n, b}\right) / 2}\left(\frac{1}{2 k}\right) M_{(1-\nu) / 2,\left(i p_{n, b}\right) / 2}\left(\frac{1}{2 b}\right) \\
& \quad+\sum_{n=1}^{N_{\nu}(b)} e^{-\left(\left(\nu^{2}-q_{n, b}^{2}\right) \tau\right) / 2} \frac{q_{n, b} \Gamma\left(\left(\nu+q_{n, b}\right) / 2\right)}{4 \eta_{n, b}^{(\nu)} \Gamma\left(1+q_{n, b}\right)}(2 k)^{(\nu+3) / 2} \\
& \quad \times e^{-1 /(4 k)} W_{-(\nu+3) / 2,\left(q_{n, b}\right) / 2}\left(\frac{1}{2 k}\right) M_{(1-\nu) / 2,\left(q_{n, b}\right) / 2}\left(\frac{1}{2 b}\right) \cdot(15)
\end{aligned}
$$

The problem of computing $P_{b}^{(\nu)}(k, \tau)$ is thus reduced to the root-finding problem. The roots $p$ and $q$ have to be determined numerically. Note that for any $\nu \in \mathbb{R}$, there is an infinite sequence of roots $p_{n, b}$ increasing to infinity, but the contributions from higher roots in the series (15) are suppressed with the factors $e^{-p_{n, b}^{2} \tau / 2}$. For $\nu<0$, there are an additional $0 \leqslant N_{\nu}(b) \leqslant[|\nu| / 2]+1$ roots $q_{n, b}$ that contribute the second (finite) sum in Equation (15) (for $\nu \geqslant 0$, there are no additional roots).

In the limit $b \uparrow \infty$, the series representation (15) for $P_{b}^{(\nu)}(k, \tau)$ converges to the integral representation (16) for $P^{(\nu)}(k, \tau)$. However, as we will see in $\S 6$, for typical values of $\nu, \tau$, and $k$ encountered in applications and $b \gg k$, $P_{b}^{(\nu)}(k, \tau)$ approximates $P^{(\nu)}(k, \tau)$ so well that numerical Asian option prices computed using the series representation agree with the prices computed using the integral representation to 10 decimals or better.

\subsection{The Spectral Representation for $P^{(\nu)}(k, \tau)$}

Proposition 2. For $\nu \in \mathbb{R}, \tau>0$, and $k>0$, in the limit $b \uparrow \infty$ the series representation (15) converges to the integral representation $([x]$ denotes the integer part of $x$; $\mathbf{1}_{\{\nu<0\}}=1(0)$ if $\left.\nu<0(\nu \geqslant 0)\right)$ :

$$
\begin{aligned}
& P^{(\nu)}(k, \tau) \\
& =\frac{1}{8 \pi^{2}} \int_{0}^{\infty} e^{-\left(\left(\nu^{2}+p^{2}\right) \tau\right) / 2}(2 k)^{(\nu+3) / 2} e^{-1 /(4 k)} W_{-(\nu+3) / 2, i p / 2}\left(\frac{1}{2 k}\right) \\
& \quad \times\left|\Gamma\left(\frac{\nu+i p}{2}\right)\right|^{2} \sinh (\pi p) p d p \\
& +\mathbf{1}_{\{\nu<0\}} \frac{1}{2 \Gamma(|\nu|)}\left\{2 k \Gamma\left(|\nu|, \frac{1}{2 k}\right)-\Gamma\left(|\nu|-1, \frac{1}{2 k}\right)\right\} \\
& +\mathbf{1}_{\{\nu<-2\}} e^{-2(|\nu|-1) \tau} \frac{(|\nu|-2)}{2 \Gamma(|\nu|)} \Gamma\left(|\nu|-2, \frac{1}{2 k}\right) \\
& +\mathbf{1}_{\{\nu<-4\}} \sum_{n=2}^{[|\nu| / 2]} e^{-2 n(|\nu|-n) \tau} \frac{(-1)^{n}(|\nu|-2 n)}{2 n(n-1) \Gamma(1+|\nu|-n)} \\
& \quad \times(2 k)^{\nu+n+1} e^{-1 /(2 k)} L_{n-2}^{(|\nu|-2 n)}\left(\frac{1}{2 k}\right) .
\end{aligned}
$$

\section{Proof of Proposition 1}

From McKean (1956) and Ito and McKean (1974, pp. 153-154; see also Linetsky 2004a), for $t>0$, the transition probability density with respect to the speed measure $\mathrm{m}(y) d y$ of the diffusion process with the infinitesimal generator (8) started at $x \in[0, b)$ and killed at $b(0$ is an entrance boundary and $b$ is a killing boundary) admits a spectral representation

$$
p_{b}(t ; x, y)=\sum_{n=1}^{\infty} e^{-\lambda_{n} \tau} \varphi_{n}(x) \varphi_{n}(y)
$$


where $\left\{\lambda_{n}, \varphi_{n}\right\}_{n=1}^{\infty}$ are eigenvalues and eigenfunctions of the Sturm-Liouville problem

$$
\begin{aligned}
-(\mathscr{G} u)(x) & \equiv-2 x^{2} u^{\prime \prime}(x)-[2(\nu+1) x+1] u^{\prime}(x) \\
& =\lambda u(x), \quad x \in(0, b),
\end{aligned}
$$

with the boundary conditions at 0 and $b(\mathfrak{s}(x)$ is the diffusion scale density given by Equation (9)):

$\lim _{x \downarrow 0} \frac{u^{\prime}(x)}{\mathfrak{s}(x)}=0$,

$u(b)=0$.

The eigenfunctions $\varphi_{n}$ form an orthonormal basis in $L^{2}([0, b], m)$ with the inner product $(f, g)=$ $\int_{0}^{b} f(x) g(x) \mathfrak{m}(x) d x$ and the norm $\|f\|^{2}=(f, f)$. For $t>0$, the spectral representation (17) converges uniformly in $x, y$ on $[0, b] \times[0, b]($ McKean 1956, Theorem 3.1).

The transformation

$z=\frac{1}{2 x}, \quad u(x)=x^{(1-\nu) / 2} e^{1 /(4 x)} v(z(x))$

reduces the ordinary differential equation (ODE) (18) to the ODE for $v(z)$ :

$v_{z z}+\left(-\frac{1}{4}+\frac{\kappa}{z}+\frac{1 / 4-\mu^{2}}{z^{2}}\right) v=0$,

$\kappa:=\frac{1-\nu}{2}, \quad \mu:=\frac{1}{2} \sqrt{\nu^{2}-2 \lambda}$.

This is Whittaker's form of the confluent hypergeometric equation (Abramowitz and Stegun 1972, p. 505; Slater 1960, p. 9; Buchholz 1969, p. 11). The Whittaker functions $W_{\kappa, \mu}(z)$ and $W_{-\kappa, \mu}\left(e^{i \pi} z\right)$ provide two solutions linearly independent for any values of $\kappa$ and $\mu$ (real or complex) with the Wronskian $e^{-i \kappa \pi}$ (Buchholz 1969, p. 25).

The solution of (18) satisfying the boundary condition (19) can be taken in the form

$\psi(x, \lambda)=(2 x)^{(1-\nu) / 2} e^{1 /(4 x)} W_{(1-\nu) / 2,(1 / 2) \sqrt{\nu^{2}-2 \lambda}}\left(\frac{1}{2 x}\right)$

Because $W_{\kappa, \mu}(z)$ is entire in $\mu \in \mathbb{C}$ for fixed $\kappa \in \mathbb{R}$ and $z>0$ and is even in $\mu$ (Slater 1960, p. 11), $\psi(x, \lambda)$ is entire in $\lambda \in \mathbb{C}$ for fixed $x>0$. This solution also satisfies the initial condition

$\psi(0, \lambda)=1$

Generally, from Theorem 4.1 in Kent (1980), for fixed $x>0$ the zeros of $\psi(x, \lambda)$ are simple and positive, form a sequence $0<\lambda_{1, x}<\lambda_{2, x}<\cdots$ with $\sum_{n=1}^{\infty} \lambda_{n, x}^{-1}<\infty$, $\psi(x, \lambda)$ can be written as a canonical product

$\psi(x, \lambda)=\prod_{n=1}^{\infty}\left(1-\frac{\lambda}{\lambda_{n, x}}\right)$ and $\lambda_{n, x}>\lambda_{n, y}$ for $0<x<y$. From Ito and McKean (1974, p. 154, Equation (31)), $|\psi(x, \lambda)|<e^{\operatorname{constant} \sqrt{\lambda}}$ as $\lambda \uparrow \infty$ uniformly in $x$ on $[0, b]$.

The eigenfunctions should satisfy the boundary condition (20) at $b$. Hence, the eigenvalues $\lambda_{n}=\lambda_{n, b}$ are the (simple and positive) zeros of $\psi(b, \lambda)$. We will consider the two cases separately: $\lambda>\nu^{2} / 2$ and $0<\lambda \leqslant \nu^{2} / 2$ (for $\nu \neq 0$ ).

In the latter case, without loss of generality set $\lambda=$ $\left(\nu^{2}-q^{2}\right) / 2,0 \leqslant q<|\nu|$. Let $N_{\nu}(b) \geqslant 0$ be the number of roots of Equation (13) in the interval $0 \leqslant q<|\nu|$. Then, for $N_{\nu}(b)>0$, the eigenvalues corresponding to the roots $\left\{q_{n, b}\right.$, $\left.n=1, \ldots, N_{\nu}(b)\right\}$ (we number the roots $q$ in descending order) are $\left\{\lambda_{n, b}=\left(\nu^{2}-q_{n, b}^{2}\right) / 2, n=1, \ldots, N_{\nu}(b)\right\}$ (the eigenvalues are always numbered in ascending order). To get precise numerical values of $q_{n, b}$, we need to find the roots of Equation (13) numerically. However, for large $b$ we can get estimates by using the Whittaker function asymptotics for $\mu>0$ and $z>0$ :

$$
W_{\kappa, \mu}(z) \sim \frac{\Gamma(2 \mu)}{\Gamma(1 / 2+\mu-\kappa)} z^{1 / 2-\mu} e^{-z / 2} \quad \text { as } z \rightarrow 0 .
$$

This gives, for large $b$,

$$
\begin{aligned}
\psi(b, & \left.\left(\nu^{2}-q^{2}\right) / 2\right) \\
& =(2 b)^{(1-\nu) / 2} e^{1 /(4 b)} W_{(1-\nu) / 2, q / 2}\left(\frac{1}{2 b}\right) \\
& \sim \frac{\Gamma(q)}{\Gamma((\nu+q) / 2)}\left(\frac{1}{2 b}\right)^{(\nu-q) / 2} .
\end{aligned}
$$

For $\nu<0$, the reciprocal of the gamma function $1 / \Gamma((\nu+q) / 2)$ has zeros $(\nu+q) / 2=-n+1, n=$ $1, \ldots,[|\nu| / 2]+1$. Thus, for $\nu<0$ and large enough $b$, the total number of zeros in $[0,|\nu|)$ is equal to $N_{\nu}(b)=$ $[|\nu| / 2]+1$ and

$$
q_{n, b}=(|\nu|-2 n+2)+o(1), \quad n=1, \ldots,[|\nu| / 2]+1 .
$$

Furthermore, because $\lambda_{n, a}>\lambda_{n, b}$ for $a<b, N_{\nu}(b) \leqslant$ $[|\nu| / 2]+1$ for all $b>0$. For $\nu>0$, the right-hand side of Equation (27) never vanishes for any value of $q \in[0, \nu)$ and, hence, for large enough $b$ we have $N_{\nu}(b) \equiv 0$. Again, because $\lambda_{n, a}>\lambda_{n, b}$ for $a<b$, we conclude that, in fact, $N_{\nu}(b) \equiv 0$ for all $b>0$.

Now consider the case $\lambda>\nu^{2} / 2$. Without loss of generality, set $\lambda=\left(\nu^{2}+p^{2}\right) / 2, p>0$. Then, the eigenvalues above $\nu^{2} / 2$ are given by $\left\{\lambda_{N_{\nu}(b)+n, b}=\left(\nu^{2}+p_{n, b}^{2}\right) / 2, n=\right.$ $1,2, \ldots\}$, where $\left\{p_{n, b}, n=1,2, \ldots\right\}$ are the simple positive roots of Equation (11). To get precise numerical values of $p_{n, b}$, we need to find the roots of Equation (11) numerically. However, we can obtain estimates that improve with increasing $n$ by using the following estimate of the Whittaker function with purely imaginary second index 
$\mu=i \rho, \rho>0, \kappa \in \mathbb{R}$, and fixed $z>0$ (Shrivastava et al. 1998, Equation (3.37); Yakubovich 1996, Theorem 1.11):

$$
\begin{aligned}
W_{\kappa, i \rho}(z)= & \sqrt{2 z} e^{-\pi \rho / 2} \rho^{\kappa-(1 / 2)} \\
& \times \cos \left(\rho \ln \left(\frac{z}{4 \rho}\right)+\rho-\left(\kappa-\frac{1}{2}\right) \frac{\pi}{2}\right) \\
& \times\left[1+O\left(\rho^{-1}\right)\right] .
\end{aligned}
$$

From (29) we can estimate $p_{n, b}$ as solutions $\tilde{p}_{n, b}$ of

$\tilde{p}_{n, b}\left[\ln \left(4 b \tilde{p}_{n, b}\right)-1\right]=2 \pi\left(n+\frac{\nu}{4}-\frac{1}{2}\right)$.

This estimate turns out to be quite accurate even for small $n$ (see §6). With the view towards passing to the limit $b \uparrow \infty$, we note that for large $b$ and fixed $n$

$p_{n+1, b}-p_{n, b}=\frac{2 \pi}{\ln (b)}+o\left(\frac{1}{\ln (b)}\right)$.

As $b$ increases, $p_{n, b}$ are distributed more and more densely on the positive real line, and the corresponding eigenvalues are distributed more and more densely in $\left(\nu^{2} / 2, \infty\right)$.

The nonnormalized eigenfunctions corresponding to the eigenvalues $\left\{\lambda_{N_{\nu}(b)+n, b}=\left(\nu^{2}+p_{n, b}^{2}\right) / 2, n=1,2, \ldots\right\}$ can be taken in the form

$$
\begin{array}{r}
\psi\left(x, \lambda_{N_{\nu}(b)+n, b}\right)=(2 x)^{(1-\nu) / 2} e^{1 /(4 x)} W_{(1-\nu) / 2,\left(i p_{n, b}\right) / 2}\left(\frac{1}{2 x}\right) \\
n=1,2, \ldots
\end{array}
$$

The norms of these nonnormalized eigenfunctions are given by

$$
\begin{array}{r}
\frac{1}{\left\|\psi\left(\cdot, \lambda_{N_{\nu}(b)+n, b}\right)\right\|^{2}} \\
=\frac{2^{\nu} p_{n, b} \Gamma\left(\frac{1}{2}\left(\nu+i p_{n, b}\right)\right)}{\Gamma\left(1+i p_{n, b}\right) \xi_{n, b}^{(\nu)}} M_{(1-\nu) / 2,\left(i p_{n, b}\right) / 2}\left(\frac{1}{2 b}\right), \\
n=1,2, \ldots
\end{array}
$$

For $\nu<0$ and $N_{\nu}(b)>0$, the nonnormalized eigenfunctions corresponding to the $N_{\nu}(b)$ additional eigenvalues can be taken in the form

$$
\begin{array}{r}
\psi\left(x, \lambda_{n, b}\right)=(2 x)^{(1-\nu) / 2} e^{1 /(4 x)} W_{(1-\nu) / 2,\left(q_{n, b}\right) / 2}\left(\frac{1}{2 x}\right) \\
n=1, \ldots, N_{\nu}(b) .
\end{array}
$$

The norms of these nonnormalized eigenfunctions are given by

$$
\begin{gathered}
\frac{1}{\left\|\psi\left(\cdot, \lambda_{n, b}\right)\right\|^{2}}=\frac{2^{\nu} q_{n, b} \Gamma\left(\frac{1}{2}\left(\nu+q_{n, b}\right)\right)}{\Gamma\left(1+q_{n, b}\right) \eta_{n, b}^{(\nu)}} M_{(1-\nu) / 2,\left(q_{n, b}\right) / 2}\left(\frac{1}{2 b}\right), \\
n=1, \ldots, N_{\nu}(b) .
\end{gathered}
$$

To calculate the norms (33) and (35), we follow the recipe in $\$ 5.1$ of Linetsky (2004a). For $\lambda \in \mathbb{C}$, let $\phi(x, \lambda)$ be the solution of the ODE (18) with the initial conditions at $b$ (prime denotes differentiation in $x$ ):

$\phi(b, \lambda)=0, \quad \phi^{\prime}(b, \lambda)=-1$.

For fixed $x$, it is an entire function of $\lambda \in \mathbb{C}$. This solution is obtained in the form (see Davydov and Linetsky 2003, Equation (69))

$$
\begin{aligned}
& \phi(x, \lambda) \\
& =2 b^{2}\left(\frac{x}{b}\right)^{(1-\nu) / 2} e^{1 /(4 x)-1 /(4 b)} \frac{\Gamma\left(\nu / 2+(1 / 2) \sqrt{\nu^{2}-2 \lambda}\right)}{\Gamma\left(1+\sqrt{\nu^{2}-2 \lambda}\right)} \\
& \quad \times\left\{W_{(1-\nu) / 2,(1 / 2) \sqrt{\nu^{2}-2 \lambda}}\left(\frac{1}{2 b}\right) M_{(1-\nu) / 2,(1 / 2) \sqrt{\nu^{2}-2 \lambda}}\left(\frac{1}{2 x}\right)\right. \\
& \left.\quad-M_{(1-\nu) / 2,(1 / 2) \sqrt{\nu^{2}-2 \lambda}}\left(\frac{1}{2 b}\right) W_{(1-\nu) / 2,(1 / 2) \sqrt{\nu^{2}-2 \lambda}}\left(\frac{1}{2 x}\right)\right\} .
\end{aligned}
$$

For arbitrary $\lambda \in \mathbb{C}$, the two solutions $\psi(x, \lambda)$ and $\phi(x, \lambda)$ are generally linearly independent and their Wronskian is $(\mathfrak{s}(s)$ is the scale density (9))

$$
\begin{aligned}
w(\lambda) & :=\psi(x, \lambda) \frac{\phi^{\prime}(x, \lambda)}{\mathfrak{s}(x)}-\phi(x, \lambda) \frac{\psi^{\prime}(x)}{\mathfrak{S}(x)} \\
& =-2^{(1-\nu) / 2} b^{(\nu+3) / 2} e^{-1 /(4 b)} W_{(1-\nu) / 2,(1 / 2) \sqrt{\nu^{2}-2 \lambda}}\left(\frac{1}{2 b}\right) .
\end{aligned}
$$

While $\phi(x, \lambda)$ satisfies the boundary condition (20) at $b$ for any complex $\lambda$, generally it does not satisfy the boundary condition (19) at 0 . Only when $\lambda$ is equal to an eigenvalue $\lambda_{n, b}$, the solutions $\phi(x, \lambda)$ and $\psi(x, \lambda)$ become linearly dependent (their Wronskian (38) vanishes for $\lambda=\lambda_{n}$ ), satisfy both boundary conditions at 0 and $b$, and $\phi\left(x, \lambda_{n, b}\right)=$ $A_{n, b} \psi\left(x, \lambda_{n, b}\right)$, where the (nonzero) constant $A_{n, b}$ is

$$
\begin{aligned}
A_{n, b}= & -\frac{1}{2}(2 b)^{(\nu+3) / 2} e^{-1 /(4 b)} \frac{\Gamma\left(\nu / 2+(1 / 2) \sqrt{\nu^{2}-2 \lambda_{n, b}}\right)}{\Gamma\left(1+\sqrt{\nu^{2}-2 \lambda_{n, b}}\right)} \\
& \times M_{(1-\nu) / 2,(1 / 2) \sqrt{\nu^{2}-2 \lambda_{n, b}}}\left(\frac{1}{2 b}\right) .
\end{aligned}
$$

Finally, from Lemma 2 in Linetsky (2002) we have the norms

$$
\left\|\psi\left(\cdot, \lambda_{n, b}\right)\right\|^{2}=\frac{w^{\prime}\left(\lambda_{n, b}\right)}{A_{n, b}}, \quad w^{\prime}\left(\lambda_{n, b}\right):=\left.\frac{d w(\lambda)}{d \lambda}\right|_{\lambda=\lambda_{n, b}} .
$$

Substituting Equations (38), (39), (12), and (14) into Equation (40) gives Equations (33) and (35) for the norms. The normalized eigenfunctions can be taken in the form $\varphi_{n}(x)=\psi\left(x, \lambda_{n, b}\right) /\left\|\psi\left(\cdot, \lambda_{n, b}\right)\right\|$ and, for $t>0$, the spectral 
representation for the transition probability density takes the form

$p_{b}(t ; x, y)=\sum_{n=1}^{\infty} e^{-\lambda_{n, b} t} \frac{\psi\left(x, \lambda_{n, b}\right) \psi\left(y, \lambda_{n, b}\right)}{\left\|\psi\left(\cdot, \lambda_{n, b}\right)\right\|^{2}}$,

and the series converges uniformly in $x, y$ on $[0, b] \times[0, b]$.

Noting that $(k-x)^{+} \in L^{2}([0, b], \mathfrak{m})$ and recalling Equation (24), we have the spectral representation

$$
\begin{aligned}
& P_{b}^{(\nu)}(k, \tau)=\int_{0}^{k}(k-y) p_{b}(\tau ; 0, y) \mathfrak{m}(y) d y \\
& \quad=\sum_{n=1}^{\infty} e^{-\lambda_{n, b} \tau} \frac{c\left(\lambda_{n, b}\right)}{\left\|\psi\left(\cdot, \lambda_{n, b}\right)\right\|^{2}} \\
& c(\lambda)=\int_{0}^{k}(k-y) \psi(y, \lambda) \mathfrak{m}(y) d y \\
& =2^{-(\nu+1) / 2} k^{(\nu+3) / 2} e^{-1 /(4 k)} W_{-(\nu+3) / 2,(1 / 2) \sqrt{\nu^{2}-2 \lambda}}\left(\frac{1}{2 k}\right)
\end{aligned}
$$

To calculate the integral in (43), we used the integral identity for the Whittaker function (valid for $\mu \in \mathbb{C}, \nu \in \mathbb{R}$, $k>0$; Gradshteyn and Ryzhik 1994, p. 867, Equation (7))

$$
\begin{gathered}
\int_{0}^{k}(k-x) x^{(\nu-1) / 2} e^{-1 /(4 x)} W_{(1-\nu) / 2, \mu}\left(\frac{1}{2 x}\right) d x \\
=k^{(\nu+3) / 2} e^{-1 /(4 k)} W_{-(\nu+3) / 2, \mu}\left(\frac{1}{2 k}\right) .
\end{gathered}
$$

Substituting the ingredients in Equation (42) yields the series representation (15).

\section{Proof of Proposition 2}

The spectral representation for the transition probability density $p(t ; x, y)$ of the diffusion on $[0, \infty)$ can be obtained in the limit $b \uparrow \infty$ (McKean 1956; Linetsky 2004a, §5.2); here and in McKean (1956) the transition probability density is with respect to the speed measure, while in Linetsky (2002) it is with respect to the Lebesgue measure. Introduce a nondecreasing right-continuous jump function (the spectral function of the Sturm-Liouville problem (18)-(20)):

$\rho_{b}(\lambda):=\sum_{n=1}^{\infty} \frac{1}{\left\|\psi\left(\cdot, \lambda_{n, b}\right)\right\|^{2}} \mathbf{1}_{\left\{\lambda_{n, b} \leqslant \lambda\right\}}$,

where $\mathbf{1}_{\left\{\lambda_{n, b} \leqslant \lambda\right\}}=1$ (0) if $\lambda_{n, b} \leqslant \lambda\left(\lambda_{n, b}>\lambda\right)$. It jumps by $1 /\left\|\psi\left(\cdot, \lambda_{n, b}\right)\right\|^{2}$ at an eigenvalue $\lambda=\lambda_{n, b}$. The spectral representations (41)-(42) can be rewritten as a Stieltjes integral:

$$
\begin{aligned}
& p_{b}(t ; x, y)=\int_{[0, \infty)} e^{-\lambda t} \psi(x, \lambda) \psi(y, \lambda) d \rho_{b}(\lambda), \\
& P_{b}^{(\nu)}(k, \tau)=\int_{[0, \infty)} e^{-\lambda \tau} c(\lambda) d \rho_{b}(\lambda) .
\end{aligned}
$$

The limit $\lim _{b \uparrow \infty} \rho_{b}(\lambda)=\rho(\lambda)$ produces a nondecreasing right-continuous function-the spectral function of the Sturm-Liouville problem on $[0, \infty)$ with the boundary condition (19) at 0 . Infinity is a natural boundary for the diffusion. Applying the classification of natural boundaries in Linetsky (2002), it is oscillatory for $\lambda>\nu^{2} / 2$ and nonoscillatory for $\lambda \leqslant \nu^{2} / 2$. In other words, every solution of the Sturm-Liouville Equation (18) with $\lambda>\nu^{2} / 2$ $\left(\lambda \leqslant \nu^{2} / 2\right)$ has an infinite (finite) number of zeros in $[c, \infty)$ for any $c>0$. From the spectral classification in Linetsky (2004a), this problem has an absolutely continuous spectrum $\left[\nu^{2} / 2, \infty\right)$ plus a finite set of eigenvalues in $\left[0, \nu^{2} / 2\right]$ (it may be empty).

Spectral representations for the transition probability density $p(t ; x, y)$ and the function $P^{(\nu)}(k, \tau)$ are obtained from Equation (46) by letting $b \uparrow \infty$ :

$$
\begin{aligned}
& p(t ; x, y)=\int_{[0, \infty)} e^{-\lambda t} \psi(x, \lambda) \psi(y, \lambda) d \rho(\lambda), \\
& P^{(\nu)}(k, \tau)=\int_{[0, \infty)} e^{-\lambda \tau} c(\lambda) d \rho(\lambda) .
\end{aligned}
$$

Because the problem has an absolutely continuous spectrum $\left[\nu^{2} / 2, \infty\right)$ plus a finite set of eigenvalues in $\left[0, \nu^{2} / 2\right]$, the spectral function can be written in the form

$\rho(\lambda)=\sum_{n} \frac{1}{\left\|\psi\left(\cdot, \lambda_{n}\right)\right\|^{2}} \mathbf{1}_{\left\{\lambda \geqslant \lambda_{n}\right\}}+\rho_{a c}(\lambda) \mathbf{1}_{\left\{\lambda \geqslant \nu^{2} / 2\right\}}$,

where the sum is over the eigenvalues (if any) in $\left[0, \nu^{2} / 2\right]$ and $\rho_{a c}(\lambda)$ is absolutely continuous on $\left[\nu^{2} / 2, \infty\right)$. Roughly speaking, the discrete spectrum above $\nu^{2} / 2$ of the problem with the killing boundary at $b$ merges into the continuous spectrum of the problem on $[0, \infty)$ in the limit $b \uparrow \infty$ (as $b$ increases, eigenvalues are distributed more and more densely in $\left[\nu^{2} / 2, \infty\right)$ and in the limit merge to form a continuous spectrum).

We shall now show that the spectral function $\rho(\lambda)=$ $\lim _{b \uparrow \infty} \rho_{b}(\lambda)$ takes the form

$$
\begin{aligned}
\rho(\lambda)= & \mathbf{1}_{\{\nu<0\}} \sum_{n=0}^{[|\nu| / 2]} \frac{4(|\nu|-2 n)}{\Gamma(1+|\nu|-n) n !} \mathbf{1}_{\{\lambda \geqslant 2 n(|\nu|-n)\}} \\
& +\mathbf{1}_{\left\{\lambda \geqslant \nu^{2} / 2\right\}} \frac{1}{\pi^{2}} \int_{0}^{\sqrt{2 \lambda-\nu^{2}}}\left|\Gamma\left(\frac{\nu+i p}{2}\right)\right|^{2} \sinh (\pi p) p d p .
\end{aligned}
$$

First, for $\nu<0$ consider the eigenvalues $\left\{\lambda_{n, b}, n=1\right.$, $\left.\ldots, N_{\nu}(b)\right\}$ and the corresponding eigenfunctions (34). From Equation (28) we have (it is more convenient to label eigenvalues of the problem on $[0, \infty)$ starting with $n=0$ instead of $n=1$ as we did for the problem on $[0, b]$ )

$$
\begin{gathered}
\lambda_{n}=\lim _{b \uparrow \infty} \lambda_{n+1, b}=2 n(|\nu|-n), \quad n=0, \ldots,[|\nu| / 2], \\
\psi\left(x, \lambda_{n}\right)=(2 x)^{(1-\nu) / 2} e^{1 /(4 x)} W_{(1-\nu) / 2,-\nu / 2-n}\left(\frac{1}{2 x}\right) \\
=(-1)^{n} n !(2 x)^{n} L_{n}^{(|\nu|-2 n)}\left(\frac{1}{2 x}\right) .
\end{gathered}
$$


The last equality in (51) follows from the fact that when $\kappa=\mu+n+1 / 2$ (for integer $n$ ), the Whittaker function $W_{\kappa, \mu}(z)$ reduces to the generalized Laguerre polynomial (Buchholz 1969, p. 214)

$W_{\mu+n+1 / 2, \mu}(z)=(-1)^{n} n ! e^{-z / 2} z^{\mu+1 / 2} L_{n}^{(2 \mu)}(z)$

The norm of $\psi\left(x, \lambda_{n}\right)$ is computed using the following integral for $a>0$ (Prudnikov et al. 1986, p. 479, Equation (17); note that this equation in Prudnikov et al. has a typo-the factor $(-1)^{n}$ should not be included):

$\int_{0}^{\infty} z^{a-1} e^{-z}\left(L_{n}^{(a)}(z)\right)^{2} d z=\frac{\Gamma(1+a+n)}{n ! a}$.

The result is

$$
\frac{1}{\left\|\phi\left(\cdot, \lambda_{n}\right)\right\|^{2}}=\frac{4(|\nu|-2 n)}{\Gamma(1+|\nu|-n) n !}, \quad n=0, \ldots,\left[\frac{|\nu|}{2}\right]
$$

For $\nu<0$, this gives the sum in Equation (49).

Let $b$ be large enough so that $N_{\nu}(b)=[|\nu| / 2]+1=: N_{\nu}$. Consider the part of the spectral function (45) with the eigenvalues $\left\{\lambda_{n, b}, n=N_{\nu}+1, N_{\nu}+2, \ldots\right\}$ (to lighten notation, we omit the superscript $b$ in $\lambda_{n, b}$ and $\left.p_{n, b}\right)$ :

$$
\begin{aligned}
\sum_{n=N_{\nu}+1}^{\infty} \frac{1}{\left\|\psi\left(\cdot, \lambda_{n}\right)\right\|^{2}} \mathbf{1}_{\left\{\lambda_{n} \leqslant \lambda\right\}}= & \sum_{n=1}^{\infty} \frac{1}{\left(p_{n+1}-p_{n}\right)\left\|\psi\left(\cdot, \lambda_{N_{\nu}+n}\right)\right\|^{2}} \\
& \times \mathbf{1}_{\left\{p_{n} \leqslant \sqrt{\left.2 \lambda-\nu^{2}\right\}}\right.}\left(p_{n+1}-p_{n}\right), \quad
\end{aligned}
$$

where we divided and multiplied by $\left(p_{n+1}-p_{n}\right)$ and used $\lambda_{n}=\left(\nu^{2}+p_{n}^{2}\right) / 2$. The norms are given by Equation (33). We now show that for large $b$,

$$
\begin{aligned}
& \frac{1}{\left(p_{n+1}-p_{n}\right)\left\|\psi\left(\cdot, \lambda_{N_{\nu}+n}\right)\right\|^{2}} \\
& \quad=\frac{1}{\pi^{2}}\left|\Gamma\left(\frac{\nu+i p_{n}}{2}\right)\right|^{2} \sinh \left(\pi p_{n}\right) p_{n}+O\left(\frac{1}{\ln (b)}\right) .
\end{aligned}
$$

For small values of the argument we have (Slater 1960, Buchholz 1969; Equations (57) and (58) follow from the confluent hypergeometric series $\left.{ }_{1} F_{1}(a, b, z)\right)$

$$
\begin{aligned}
& M_{\kappa, \mu}(z)= z^{1 / 2+\mu} e^{-z / 2}[1+O(z)] \\
& W_{\kappa, \mu}(z)=\frac{\pi}{\sin (2 \mu \pi)}\left\{\frac{z^{1 / 2-\mu} e^{-z / 2}[1+O(z)]}{\Gamma(1-2 \mu) \Gamma(1 / 2+\mu-\kappa)}\right. \\
&\left.\quad-\frac{z^{1 / 2+\mu} e^{-z / 2}[1+O(z)]}{\Gamma(1+2 \mu) \Gamma(1 / 2-\mu-\kappa)}\right\} .
\end{aligned}
$$

Thus, for the Whittaker function $M$ in the numerator of Equation (33) we have, for large $b$,

$$
M_{(1-\nu) / 2,\left(i p_{n}\right) / 2}\left(\frac{1}{2 b}\right)=\left(\frac{1}{2 b}\right)^{\left(1+i p_{n}\right) / 2}\left[1+O\left(\frac{1}{2 b}\right)\right] .
$$

Now consider the denominator in (33). From Equation (58) we have, for large $b$,

$$
\begin{aligned}
& W_{(1-\nu) / 2, i p / 2}\left(\frac{1}{2 b}\right) \\
& =\frac{-i \pi}{\sinh (\pi p)}\left\{\frac{(1 /(2 b))^{(1-i p) / 2}[1+O(1 /(2 b))]}{\Gamma(1-i p) \Gamma((\nu+i p) / 2)}\right. \\
& \left.-\frac{(1 /(2 b))^{(1+i p) / 2}[1+O(1 /(2 b))]}{\Gamma(1+i p) \Gamma((\nu-i p) / 2)}\right\},
\end{aligned}
$$

and for the derivative with respect to the second index parameter

$$
\begin{aligned}
& \frac{\partial}{\partial p} W_{(1-\nu) / 2, i p / 2}\left(\frac{1}{2 b}\right) \\
& =\left(\frac{1}{2 b}\right)^{(1-i p) / 2}\left\{\frac{\pi \ln (2 b)}{2 \sinh (\pi p) \Gamma(1-i p) \Gamma((\nu+i p) / 2)}+O(1)\right\} \\
& +\left(\frac{1}{2 b}\right)^{(1+i p) / 2}\left\{\frac{\pi \ln (2 b)}{2 \sinh (\pi p) \Gamma(1+i p) \Gamma((\nu-i p) / 2)}\right. \\
& +O(1)\} .
\end{aligned}
$$

We now evaluate it at $p=p_{n}$. Because at $p=p_{n}$ the Whittaker function vanishes,

$W_{(1-\nu) / 2,\left(i p_{n}\right) / 2}\left(\frac{1}{2 b}\right)=0$;

using Equation (60) we have

$$
\begin{aligned}
& \left.\frac{\partial}{\partial p} W_{(1-\nu) / 2,(i p) / 2}\left(\frac{1}{2 b}\right)\right|_{p=p_{n, b}} \\
& =\left(\frac{1}{2 b}\right)^{\left(1+i p_{n}\right) / 2} \\
& \quad \times\left\{\frac{\pi \ln (2 b)}{\sinh \left(\pi p_{n}\right) \Gamma\left(1+i p_{n}\right) \Gamma\left(\left(\nu-i p_{n}\right) / 2\right)}+O(1)\right\} .
\end{aligned}
$$

Putting together (31), (33), (59), and (62), we arrive at (56). Substituting the result (56) into (55) and passing to the limit $b \uparrow \infty$ (by Equation (31), $\left(p_{n+1, b}-p_{n, b}\right) \rightarrow 0$ as $b \uparrow \infty$ ), we obtain the integral term in Equation (49).

The spectral expansion for $p(t ; x, y)$ is given by Equation (47) with the spectral function (49). We now recall the identity in law (7) where $X$ is the diffusion started at 0 and obtain the probability density $p_{A_{\tau}^{(\nu)}}(y)=p(\tau ; 0, y) \mathfrak{m}(y)$ for the Brownian exponential functional $A_{\tau}^{(\nu)}(\tau>0, \nu \in \mathbb{R})$ :

$$
\begin{aligned}
p_{A_{\tau}^{(\nu)}}(y)= & \frac{1}{2 \pi^{2}} \int_{0}^{\infty} e^{-\left(\left(\nu^{2}+p^{2}\right) \tau\right) / 2}(2 y)^{(\nu-1) / 2} e^{-1 /(4 y)} \\
& \times W_{(1-\nu) / 2, i p / 2}\left(\frac{1}{2 y}\right)\left|\Gamma\left(\frac{\nu+i p}{2}\right)\right|^{2} \sinh (\pi p) p d p
\end{aligned}
$$




$$
\begin{aligned}
& +\mathbf{1}_{\{\nu<0\}} \sum_{n=0}^{[|\nu| / 2]} e^{-2 n(|\nu|-n) \tau} \frac{(-1)^{n} 2(|\nu|-2 n)}{\Gamma(1+|\nu|-n)} \\
& \times(2 y)^{n-1-|\nu|} e^{-1 /(2 y)} L_{n}^{(|\nu|-2 n)}\left(\frac{1}{2 y}\right) .
\end{aligned}
$$

Furthermore, substituting the spectral function in the spectral expansion (47) for $P^{(\nu)}(k, \tau)$, recalling the result (43) for the coefficients and, for $\nu<0$, using Equation (52) and

$$
W_{\mu-1 / 2, \mu}(z)=z^{1 / 2-\mu} e^{z / 2} \Gamma(2 \mu, z)
$$

(Buchholz 1969, p. 209) to simplify the terms in the sum, we obtain the result (16). It can also be obtained by directly calculating the integral $\int_{0}^{k}(k-y) p_{A_{\tau}^{(\nu)}}(y) d y$ with the density (63).

Remarks on the Related Literature. For $\nu<0$, the spectral representation for a diffusion $X$ on $[0, \infty)$ started at $X_{0}=x \geqslant 0$, and with the infinitesimal generator (8) first appeared in Wong (1964, Equation (38)). The density (63) is a particular case of Wong's result for the process started at zero, $x=0$. Schenzle and Brand (1979) obtained a similar result for a diffusion $Y$ with the infinitesimal generator

$(\mathscr{G} f)(x)=\frac{1}{2} a^{2} x^{2} f^{\prime \prime}(x)+\left(b-c x^{2}\right) x f^{\prime}(x)$.

Monthus and Comtet (1994) observed that, for $a=1, b=$ $1 / 2-\nu$, and $c=1 / 2$, Schenzle and Brand's diffusion is a reciprocal square root of Wong's diffusion, $Y=X^{-1 / 2}$ and, thus, rediscovered Wong's spectral representation from that of Schenzle and Brand. Comtet and Monthus (1996) and Comtet et al. (1998) used this spectral representation to study Brownian exponential functionals.

The Liouville transformation

$z=\frac{1}{\sqrt{2}} \ln x, \quad u(x)=x^{-\nu / 2} e^{1 /(4 x)} v(z(x))$

reduces the Sturm-Liouville equation (19) associated with the diffusion infinitesimal generator (8) to the Schrödinger equation with Morse (1929) potential:

$-v^{\prime \prime}(z)+\left(\frac{1}{8} e^{-2 \sqrt{2} z}+\frac{1}{2}(\nu-1) e^{-\sqrt{2} z}+\frac{\nu^{2}}{2}\right) v(z)=\lambda v(z)$

Morse (1929) has obtained the discrete part of the spectral representation, but not the absolutely continuous part. Another interesting connection is with Brownian motion on the hyperbolic plane in a magnetic field (Grosche 1988, Ikeda and Matsumoto 1999). We also note a close connection to the problem of pricing options on assets continuously paying cash dividends (Lipton 1999; Lewis 1998, 2002).
Remarks on Approximating $P^{(\nu)}(k, \tau)$ with $P_{b}^{(\nu)}(k, \tau)$ for Finite $b$. In this paper, our approach is to first construct the spectral representation on a finite interval and then take the limit $b \uparrow \infty$. The problem on the finite interval has a purely discrete spectrum, and the resulting spectral representation of $P_{b}^{(\nu)}(k, \tau)$ does not require any numerical integration. In the limit $b \uparrow \infty$ we recover the spectral representation of $P^{(\nu)}(k, \tau)$ that includes an integral over the absolutely continuous part of the spectrum plus, for $\nu<0$, a sum over the eigenvalues. The benefit of this approach is that we end up with two representations-the series representation and the integral representation. The series has the advantage that it does not require any numerical integrations.

We can derive the following upper bound for the approximation error $\left(M_{t}\right.$ is the maximum of diffusion $X$ started at 0 on the time interval $[0, t])$ :

$$
\begin{aligned}
P^{(\nu)}(k, \tau)-P_{b}^{(\nu)}(k, \tau) & =\mathbb{E}\left[\mathbf{1}_{\left\{\mathscr{I}_{b} \leqslant \tau\right\}}\left(k-X_{\tau}\right)^{+}\right] \\
& \leqslant k \mathbb{P}\left(M_{\tau} \geqslant b\right) \leqslant \frac{k}{b} \mathbb{E}\left[M_{\tau}\right] .
\end{aligned}
$$

This bound is linear in the ratio $k / b$. Selecting $b$ large enough relative to $k$, we can make the error arbitrarily small. For $\nu \geqslant-1, X$ is a nonnegative submartingale and we have the maximal inequality: $\mathbb{P}\left(M_{\tau} \geqslant b\right) \leqslant \mathbb{E}\left[X_{\tau}\right] / b$. From the representation $X_{t}=e^{2\left(B_{t}+\nu t\right)} \int_{0}^{t} e^{-2\left(B_{u}+\nu u\right)} d u$ and Fubini's theorem, we have $\mathbb{E}\left[X_{\tau}\right]=(1 /(2(\nu+1)))\left(e^{2(\nu+1) \tau}-1\right)$ for $\nu>-1$ and $\mathbb{E}\left[X_{\tau}\right]=\tau$ for $\nu=0$. This gives us a more explicit error bound:

$$
\begin{aligned}
& P^{(\nu)}(k, \tau)-P_{b}^{(\nu)}(k, \tau) \\
& \leqslant \frac{\tau k}{b} \begin{cases}(1 /(2(\nu+1) \tau))\left(e^{2(\nu+1) \tau}-1\right), & \nu>-1, \\
1, & \nu=-1 .\end{cases}
\end{aligned}
$$

These bounds are very conservative, and actual errors turn out to be orders of magnitude smaller.

\section{Computational Results}

In this section, we use the series representation (15) and the integral representation (16) to compute Asian option prices. We compute Asian put prices first, then use the callput parity (3) to compute call prices. We consider seven combinations of parameters given in Table 1. These combinations of parameters have been used as test cases for various numerical methods by Eydeland and Geman (1995), Fu et al. (1998), Shaw (1997, 2002), Craddock et al. (2000), Dufresne (2000), and Vecer $(2001,2002)$. Table 1 gives the interest rate $r$, volatility $\sigma$, time to expiration $T$, and the initial asset price $S_{0}$. It is assumed that the underlying asset pays no dividends $(q=0)$, the strike price is $K=2.0$ for all cases, and all options start at time zero, $t=0$ (newly written contracts). The standardized parameters $\nu, \tau$, and $k$ are computed using Equation (6). 
Table 1. Asian option parameter values.

\begin{tabular}{llcccccl}
\hline Case & \multicolumn{1}{c}{$r$} & $\sigma$ & $T$ & $S_{0}$ & $\nu$ & \multicolumn{1}{c}{$\tau$} & \multicolumn{1}{c}{$k$} \\
\hline 1 & 0.02 & 0.10 & 1 & 2.0 & 3 & 0.0025 & 0.0025 \\
2 & 0.18 & 0.30 & 1 & 2.0 & 3 & 0.0225 & 0.0225 \\
3 & 0.0125 & 0.25 & 2 & 2.0 & -0.6 & 0.03125 & 0.03125 \\
4 & 0.05 & 0.50 & 1 & 1.9 & -0.6 & 0.0625 & 0.065789 \\
5 & 0.05 & 0.50 & 1 & 2.0 & -0.6 & 0.0625 & 0.0625 \\
6 & 0.05 & 0.50 & 1 & 2.1 & -0.6 & 0.0625 & 0.059524 \\
7 & 0.05 & 0.50 & 2 & 2.0 & -0.6 & 0.125 & 0.125 \\
\hline
\end{tabular}

Note. For all seven cases $q=0$ and $K=2.0$.

We used Mathematica 4.0 running on a Pentium III PC for all computations in this paper. Pick $b=1$ and consider the steps needed to compute the Asian put price using the series representation (15) (note that $b=1$ is much greater than the normalized strike $k$ for all seven cases in Table 1). First, we need to determine the eigenvalues. The eigenvalues need to be determined only once for all options with different strikes, times to expiration, and current values of the underlying asset price. The eigenvalues depend only on the parameter $\nu$, which is a characteristic of the underlying stochastic process, and are independent of the current value of the process or the terms of the option contract to be valued. To determine the eigenvalues, we need to numerically determine zeros of the Whittaker functions (11) and (13) with respect to the parameters $p$ and $q$ in the second index. The Whittaker functions are related to the Kummer and Tricomi confluent hypergeometric functions $M(a, b, z), U(a, b, z)$ that are provided as built-in functions in the Mathematica software package (Abramowitz and Stegun 1972, p. 505):

$$
\begin{aligned}
& M_{\kappa, \mu}(z)=z^{1 / 2+\mu} e^{-z / 2} M\left(\frac{1}{2}+\mu-\kappa, 1+2 \mu, z\right), \\
& W_{\kappa, \mu}(z)=z^{1 / 2+\mu} e^{-z / 2} U\left(\frac{1}{2}+\mu-\kappa, 1+2 \mu, z\right) .
\end{aligned}
$$

For the seven test cases in Table 1, we need to determine the eigenvalues twice for $\nu=3$ and $\nu=-0.6$. For $b=1$ and $\nu=3$ and $\nu=-0.6$, the Whittaker function (13) has no zeros in the interval $0 \leqslant q<|\nu|$ (it is strictly positive in this interval). Next, consider the Whittaker function (11). Using remarkable capabilities of Mathematica in handling special functions with arbitrary precision arithmetics, we are able to determine the first one thousand zeros of the Whittaker function (11) with the precision of two hundred significant digits in just over one hour on a PC. Thus, numerical determination of zeros of the Whittaker function does not present any computational problems and can be accomplished with arbitrary precision. Moreover, the zero finding can be accelerated by solving Equation (31) and using the solution $\tilde{p}_{n, b}$ as the first estimate of the exact zero $p_{n, b}$ in a numerical zero-finding procedure. Table 2 gives the first several zeros and the corresponding eigenvalues. The estimates $\tilde{p}_{n, b}$ are given in parentheses. One can see from the table that the estimates are already quite close to the exact zeros
Table 2. Eigenvalues for $b=1$ and $\nu=3$ and $\nu=-0.6$.

\begin{tabular}{ccc}
\hline$n$ & Zero & Eigenvalue \\
\hline$\nu=3$ & & \\
1 & $4.041095077(4.27215)$ & $12.66522471(13.6257)$ \\
2 & $6.198998995(6.33346)$ & $23.71379427(24.5563)$ \\
3 & $8.103928065(8.19967)$ & $37.33682504(38.1173)$ \\
4 & $9.875120946(9.94975)$ & $53.25900684(53.9987)$ \\
5 & $11.55802557(11.6193)$ & $71.29397758(72.0040)$ \\
10 & $19.22528830(19.2579)$ & $189.3058551(189.934)$
\end{tabular}

\begin{tabular}{lcc}
$\nu=-0.6$ & & \\
1 & $1.943531071(2.0193)$ & $2.068656513(2.21879)$ \\
2 & $4.473537623(4.49156)$ & $10.18626943(10.2671)$ \\
3 & $6.516559483(6.52695)$ & $21.41277375(21.4805)$ \\
4 & $8.371763836(8.37912)$ & $35.22321486(35.2848)$ \\
5 & $10.11419245(10.1199)$ & $51.32844445(51.3863)$ \\
\hline
\end{tabular}

for low values of $n$, and the accuracy rapidly increases with $n$. After the eigenvalues are determined, Asian put prices are calculated using the series representation (15). To compute the derivatives (12) and (14) of the Whittaker function $W_{\kappa, \mu}(z)$ with respect to its second index, recall its relationship with the Tricomi confluent hypergeometric function $U(a, b, z)$. Derivatives of the Tricomi function with respect to the first and second index are available in Mathematica with the calls Hypergeometric $\mathrm{U}^{(1,0,0)}[a, b, z]$ and Hypergeometric $\mathrm{U}^{(0,1,0)}[a, b, z]$.

The integral representation (16) can be programmed in Mathematica using the built-in numerical integration routine. The advantage of the series representation is that no numerical integration is required. We have computed Asian option prices in the seven test cases of Table 1 using both the series representation (15) and the integral representation (16). Both the series with $b=1$ and the integral gave identical results in all seven test cases to 10 decimals or better. The results for the Asian call options are reported in Table 3 in the column marked EE (Eigenfunction Expansion). The numbers in parentheses next to the option prices give the number of terms in the series (15) needed to achieve 10-decimal accuracy. One can see that while for Case 7, with the largest value of $\tau=\left(\sigma^{2} T\right) / 4$, only 13 terms are enough to achieve this level of accuracy; Case 1 with the smallest value of $\tau$ requires 400 terms. The integral representation (16) using the built-in numerical integration routine gave identical option values in all seven cases. However, it was slower than the series due to numerical integration. In both cases, the dimensionless time to expiration $\tau=\left(\sigma^{2} T\right) / 4$ is the crucial parameter that controls numerical convergence of the series (higherorder terms are suppressed with $e^{-p_{n, b}^{2} \tau / 2}$ ) and the integral $\left(e^{-p^{2} \tau / 2}\right.$ in the integral). The larger the value of $\tau$, the faster the convergence. For smaller values of this parameter, convergence significantly slows down, as one needs to integrate an oscillatory integrand over a larger range in $p$ until the factor $e^{-p^{2} \tau / 2}$ suppresses the integrand, or sum up more terms in a nearly alternating signs series until the factor $e^{-p_{n, b}^{2} \tau / 2}$ suppresses the large- $n$ terms. 
Table 3. Asian call option prices.

\begin{tabular}{llclcc}
\hline Case & EE & SLT & MC & TLB & TUB \\
\hline 1 & $0.0559860415(400)$ & 0.055986 & $0.05602(0.00017)$ & 0.055985 & 0.055989 \\
2 & $0.2183875466(57)$ & 0.218388 & $0.2185(0.00059)$ & 0.218366 & 0.218473 \\
3 & $0.1722687410(41)$ & 0.172269 & $0.1725(0.00063)$ & 0.172226 & 0.172451 \\
4 & $0.1931737903(24)$ & 0.193174 & $0.1933(0.00084)$ & 0.193060 & 0.193799 \\
5 & $0.2464156905(23)$ & 0.246416 & $0.2465(0.00095)$ & 0.246298 & 0.247054 \\
6 & $0.3062203648(23)$ & 0.306220 & $0.3064(0.00106)$ & 0.306094 & 0.306904 \\
7 & $0.3500952190(13)$ & 0.350095 & $0.3503(0.00146)$ & 0.349779 & 0.352556 \\
\hline
\end{tabular}

Note. Parameters are as in Table 1.

For comparison, Table 3 also gives Asian call-option prices reported by authors using different numerical methods. The column marked SLT gives recent numerical Laplace transform inversion results of Shaw (2002). Other authors who investigate numerical inversion of the Geman-Yor Laplace transform include Eydeland and Geman (1995), Fu et al. (1998), and Craddock et al. (2000). Shaw's implementation produces the most accurate results, agreeing with our results to six decimals. Vecer (2001, 2002) develops numerical PDE methods. Using the built-in numerical PDE solver in Mathematica, he was able to match our and Shaw's numbers to six-decimal accuracy (Vecer 2002). The column marked MC reports the values and standard errors obtained by Monte Carlo simulation in Dufresne (2000). The columns TLB and TUB give the values of lower and upper bounds for the Asian call prices computed by Giles Thompson using his method (Thompson 1999); the author thanks Giles Thompson for computing these bounds. We note that Thompson's lower bound is exceedingly accurate for small times to maturity/small volatilities.

\section{Conclusion}

In this paper we derive two analytical formulas for the value of the continuously sampled arithmetic Asian option when the underlying asset price follows geometric Brownian motion. We use an identity in law between the integral of geometric Brownian motion over a finite time interval $[0, t]$ and the state at time $t$ of a one-dimensional diffusion process with affine drift and linear diffusion, and express Asian option values in terms of spectral expansions associated with the diffusion infinitesimal generator (see Linetsky 2004a for a detailed exposition of the spectral expansion approach and Davydov and Linetsky 2003, Gorovoi and Linetsky 2004, and Linetsky 2004b for further option-pricing applications). The first formula is an infinite series of terms involving Whittaker functions $M$ and $W$. The second formula is a single real integral of an expression involving Whittaker function $W$ plus (for some parameter values) a finite number of additional terms involving incomplete gamma functions and Laguerre polynomials. The two formulas allow accurate computation of continuously sampled arithmetic Asian option prices and provide benchmarks for numerical methods such as Monte
Carlo simulation, numerical PDE schemes, and numerical Laplace transform inversion algorithms.

\section{Acknowledgments}

The author is grateful to Marc Yor for correspondence and for bringing references Carmona et al. (1997), Comtet and Monthus (1996), Comtet et al. (1998), Donati-Martin et al. (2001), Ikeda and Matsumoto (1999), and Monthus and Comtet (1994) to his attention and to the two anonymous referees for extensive comments and suggestions. This research was partially supported by the U.S. National Science Foundation under grant DMI-0200429.

\section{References}

Abramowitz, M., I. A. Stegun. 1972. Handbook of Mathematical Functions. Dover, New York.

Borodin, A. N., P. Salminen. 1996. Handbook of Brownian Motion. Birkhauser, Boston, MA.

Boyle, P. P. 1996. New life forms on the option landscape. J. Financial Engrg. 2(3) 217-252.

Boyle, P. P., D. Emanuel. 1980. Options on the general mean. Working paper, University of British Columbia, Vancouver, British Columbia, Canada.

Boyle, P. P., M. Broadie, P. Glasserman. 1997. Monte Carlo methods for security pricing. J. Econom. Dynam. Control 21 1267-1321.

Buchholz, H. 1969. The Confluent Hypergeometric Function. Springer, Berlin, Germany.

Carmona, P., F. Petit, M. Yor. 1997. On the distribution and asymptotic results for exponential functionals of Levý processes. M. Yor, ed. Exponential Functionals and Principal Values Related to Brownian Motion. Biblioteca de la Revista Matematica Iberoamericana. Madrid, Spain, 73-121.

Comtet, A., C. Monthus. 1996. Diffusion in a one-dimensional random medium and hyperbolic Brownian motion. J. Physics A 29 1331-1345.

Comtet, A., C. Monthus, M. Yor. 1998. Exponential functionals of Brownian motion and disordered systems. J. Appl. Probab. 35 255-271.

Craddock, M., D. Heath, E. Platen. 2000. Numerical inversion of Laplace transforms: A survey of techniques with applications to derivatives pricing. J. Computational Finance 4(1) 57-81.

Davydov, D., V. Linetsky. 2003. Pricing options on scalar diffusions: An eigenfunction expansion approach. Oper. Res. 51 185-209.

Donati-Martin, C., R. Ghomrasni, M. Yor. 2001. On certain Markov processes attached to exponential functionals of Brownian motion: 
Applications to Asian options. Revista Matematica Iberoamericana, Madrid 17(1) 179-193.

Dufresne, D. 1989. Weak convergence of random growth processes with applications to insurance. Insurance: Math. Econom. 8 187-201.

Dufresne, D. 1990. The distribution of a perpetuity, with applications to risk theory and pension funding. Scandinavian Actuarial J. 39-79.

Dufresne, D. 2000. Laguerre series for Asian and other options. Math. Finance 10 407-428.

Dufresne, D. 2001. The integral of geometric Brownian motion. Adv. Appl. Probab. 33 223-241.

Dunford, N., J. Schwartz. 1963. Linear Operators. Part II: Spectral Theory (Self-Adjoint Operators in Hilbert Spaces). Wiley, NJ.

Erdelyi, A. 1953. Higher Transcendental Functions, Vol. 2. McGraw-Hill, New York.

Eydeland, A., Geman, H. 1995. Domino effect. RISK 8(4) 65-67.

Falloon, W. 1998. Windows on risk. RISK (June) 42-45.

Fu, M., D. Madan, T. Wang. 1998. Pricing Asian options: A comparison of analytical and Monte Carlo methods. J. Computational Finance 2(2) 49-74.

Geman, H., M. Yor. 1993. Bessel processes, Asian options and perpetuities. Math. Finance 3 349-375.

Gorovoi, V., V. Linetsky. 2004. Black's model of interest rates as options, eigenfunction expansions and Japanese interest rates. Math. Finance 14 49-78.

Gradshteyn, I. S., I. M. Ryzhik. 1994. Tables of Integrals, Series and Products. Academic Press, New York.

Grosche, C. 1988. The path integral on the Poincaré upper half plane with a magnetic field and for the Morse potential. Ann. Phys. 187 $110-134$.

Ikeda, N., H. Matsumoto. 1999. Brownian motion on the hyperbolic plane and Selberg trace formula. J. Funct. Anal. 163 63-110.

Ito, K., H. McKean. 1974. Diffusion Processes and Their Sample Paths. Springer, Berlin, Germany.

Kemna, A., A. Vorst. 1990. A pricing method for options based on average values. J. Banking Finance 14 113-129.

Kent, J. 1980. Eigenvalue expansions for diffusion hitting times. Z. Wahrsch. verw. Geb. 52 309-319.

Lewis, A. 1998. Applications of eigenfunction expansions in continuoustime finance. Math. Finance 8 349-383.

Lewis, A. 2002. Asian connections. Wilmott Magazine (September) 57-63.

Linetsky, V. 2004a. The spectral decomposition of the option value. Internat. J. Theoretical Appl. Finance 7 337-384.

Linetsky, V. 2004b. Lookback options and diffusion times: A spectral expansion approach. Finance and Stochastics 8 373-398.

Lipton, A. 1999. Similarities via self-similarities. RISK (September) 101-105.
Marcozzi, M. D. 2003. On the valuation of Asian options by variational methods. SIAM J. Sci. Comput. 24 1124-1140.

McKean, H. 1956. Elementary solutions for certain parabolic partial differential equations. Trans. Amer. Math. Soc. 82 519-548.

Milevsky, M. A., S. E. Posner. 1998. Asian options, the sum of lognormals, and the reciprocal gamma distribution. J. Financial Quant. Anal. 33 409-422.

Monthus, C., A. Comtet. 1994. On the flux distribution in a onedimensional disordered system. J. Phys. I (France) 4 635-653.

Morse, P. M. 1929. Diatomic molecules according to the wave mechanics: II. Vibrational levels. Physical Rev. 34 57-64.

Prudnikov, A. P., Yu A. Brychkov, O. I. Marichev. 1986. Integrals and Series, Vol. 2. Gordon and Breach, New York.

Rogers, L. C. G., Z. Shi. 1995. The value of an Asian option. J. Appl. Probab. 32 1077-1088.

Schenzle, A., H. Brand. 1979. Multiplicative stochastic processes in statistical physics. Physical Rev. A 20 1628-1647.

Shaw, W. 1997. Modeling Financial Derivatives with Mathematica. Cambridge University Press, Cambridge, U.K.

Shaw, W. 2002. Pricing Asian options by contour integration, including asymptotic methods for low volatility. Working paper, Nomura, London, U.K.

Shrivastava, H. M., Yu. V. Vasil'ev, S. B. Yakubovich. 1998. A class of index transforms with Whittaker's function in the index. Quart. J. Math. Oxford 49(2) 375-394.

Slater, L. J. 1960. Confluent Hypergeometric Functions. Cambridge University Press, Cambridge, U.K.

Thompson, G. W. P. 1999. Fast narrow bounds on the value of Asian options. Working paper, Judge Institute of Management, University of Cambridge, Cambridge, U.K.

Turnbull, S., L. Wakeman. 1992. A quick algorithm for pricing European average options. J. Financial Quant. Anal. 26 377-389.

Vecer, J. 2001. A new PDE approach for pricing arithmetic average Asian options. J. Computational Finance 4(4) 105-113.

Vecer, J. 2002. Unified Asian pricing. RISK (June) 113-116.

Wong, E. 1964. The construction of a class of stationary Markoff processes. R. Bellman, ed. Sixteenth Symposium in Applied MathematicsStochastic Processes in Mathematical Physics and Engineering. American Mathematical Society, Providence, RI, 264-276.

Yakubovich, S. B. 1996. Index Transforms. World Scientific, Singapore.

Yor, M. 1992. On some exponential functionals of Brownian motion. Adv. Appl. Probab. 24 509-531.

Yor, M. 2001. Exponential Functionals of Brownian Motion and Related Processes. Springer, Berlin, Germany.

Zvan, R., P. Forsyth, K. Vetzal. 1997. Robust numerical methods for PDE models of Asian options. J. Computational Finance 1(2) 39-78. 